\title{
Responses of Rhopalosiphum padi(L.) (Homoptera: Aphididae) on Different Wheat Cultivars under Laboratory Conditions
}

\author{
F. R. La Rossa ${ }^{1^{*}}$, A. C. Giudici ${ }^{1}$, A. Vasicek ${ }^{2}$, M. C. López ${ }^{2}$ and C. Bainotti ${ }^{3}$ \\ ${ }^{1}$ Institute of Agricultural Microbiology and Zoology, Veterinary and Agronomic Sciences Research \\ Center, National Institute of Agricultural Technology, Buenos Aires, Argentina. \\ ${ }^{2}$ Department of Agricultural Zoology, Faculty of Agricultural and Forestry Sciences, National University \\ of La Plata, Buenos Aires, Argentina. \\ ${ }^{3}$ Marcos Juárez Experimental Agricultural Station, National Institute of Agricultural Technology, \\ Córdoba, Argentina.
}

Authors' contributions

This work was carried out in collaboration between all authors. All authors read and approved the final manuscript.

Article Information

DOI: $10.9734 / A J A A R / 2017 / 33775$

Editor(s):

(1) Iskender Tiryaki, Department of Agricultural Biotechnology, Faculty of Agriculture, Canakkale Onsekiz Mart University, Canakkale, Turkey. Reviewers:

(1) Mohammed Suleiman, Umaru Musa Yar'adua University, Katsina, Nigeria. (2) Julian Chen, Institute of Plant Protection in the Chinese Academy of Agricultural Sciences, China. (3) Lucy Alford, University of Glasgow, United Kingdom. Complete Peer review History: http://www.sciencedomain.org/review-history/19755

Original Research Article

Received $29^{\text {th }}$ April 2017

Accepted $25^{\text {th }}$ June 2017

Published $29^{\text {th }}$ June 2017

\section{ABSTRACT}

Bird cherry-oat aphid, Rhopalosiphum padi (L.), a polyphagous species with a worldwide distribution, is an important pest of wheat as well as the main vector of barley yellow dwarf virus. The development, survivorship, and life table parameters of R.padi were evaluated in a growth chamber on seven wheat cultivars as follows: ACA 315, Baguette 12 P, Biolnta 1002, Biolnta 2004, Buck Meteoro, Klein Yarará and LE 2330 at controlled conditions $\left(20 \pm 1{ }^{\circ} \mathrm{C}\right.$; about $70 \% \mathrm{RH}$; $14 \mathrm{~h}$ photophase). The development times of immatures ranged from 6.6 days on Buck Meteoro to 9.9 days on ACA315, whereas immature survival was 90 to $100 \%$. The intrinsic rate of increase $\left(r_{m}\right)$ for ACA 315, Baguette $12 \mathrm{P}$ and Biolnta 2004 were the highest. Jackknife estimates of $r_{m}$ ranged from 0.327 to 0.204 females/female/day on Biolnta 1002 and ACA315, respectively. The mean population generation times $(T)$ on these hosts ranged from 10.91 to 19.66 days. The

*Corresponding author: Email: larossa.francisco@inta.gob.ar; 
highest net reproductive rate $\left(\mathrm{R}_{0}\right)$ were on Biolnta 2004 (98.98 females/female/generation) and the lowest on Biolnta 1002, Buck Meteoro, Klein Yarará and LE 233 (35.32 to 39.59). Because of the high coefficient of determination (pseudo- $R^{2}$ ) values in Gompertz and Weibul models, survival data from different cultivars had a good fit to both models. The results pointed ACA 315, Baguette $12 \mathrm{P}$ and partially Biolnta 2004 as the least suitable host plants, indicating that they were the most resistant to $R$. padi among the cultivars we tested.

Keywords: Fertility life tables; intrinsic rate of increase; host plant suitability; survival models.

\section{INTRODUCTION}

Distribution of the bird cherry-oat aphid, Rhopalosiphum padi (L.) is virtually cosmopolitan and anholocyclic populations occur in warm climates or where primary hosts are unavailable [1]. R. padi attacks all the major cereals and pasture grasses and is probably the major pest of temperate cereal crops on a world scale $[2,3]$. Among the numerous aphid species found in cereals, $R$. padi is considered to be one of the major pests in South American grain-producing regions even with different climates such as the semiarid Pampas of Argentina and southern Brazil $[4,5]$. In the past, $R$. padi was considered as having low populations and sporadic occurrence on wheat [6]. However, in recent years, this aphid has become the most frequent species on wheat crop and is abundant throughout all developmental stages of wheat plants [5]. This aphid damages wheat plants both by direct feeding and as a result of virus transmission and is a main vector of Barley yellow dwarf virus (BYDV) (Luteoviridae: Luteovirus) the causal agent of one of the most serious diseases of cereals worldwide [7]. Cultural control methods involve use of agronomic practices to reduce insect pest abundance and damage in the Integrated Pest Management (IPM), plant resistance to insects refers to the use of resistant crop varieties to suppress insect pest damage [8]. The antibiosis category of plant resistance occurs when the negative effects of a resistant plant affect the biology (growth, survival and reproduction) of an arthropod attempting to use that plant as a host $[9,10]$. Life tables are powerful tools for analyzing and understanding the impact that an external factor, such as antibiosis, has upon the biology of an insect [11,12]. Biological parameters, such as the duration of developmental stages and population growth obtained from fertility life tables, are important to assess host plant resistance to aphids and other pests [13,14]. The aim of this research was to determine the effects of different wheat cultivars on the biology and demography of $R$. padi under controlled environmental conditions.

\section{MATERIALS AND METHODS}

\subsection{Aphid Colony}

The aphid colony of $R$. padi used was obtained from a greenhouse of the Instituto de Microbiología y ZoologíaAgrícola (CNIA-INTA, Castelar, Buenos Aires, Argentina, 3436' 21.6" $\mathrm{S}, 5840$ '48"W) reared from viviparous apterous females collected on spontaneous Poaceae in the field near that institute. Aphids were maintained on unknown but susceptible wheat cultivar in the greenhouse (about ${ }^{2} 3^{\circ} \mathrm{C} ; 12 \mathrm{~h}$ photophase).

\subsection{Host Plants}

Seven commercial wheat (Triticum aestivum) cultivars that are commonly grown in wheatproducing regions of Argentina, including ACA 315, Baguette $12 \mathrm{P}$, Biolnta 1002, Biolnta 2004, Buck Meteoro, Klein Yarará and LE 2330 were used. Due to its intermediate or long cycle and therefore susceptibility to aphids attack, these cultivars were chosen. All plants were obtained from seeds and maintained in a growth chamber in cylindrical plastic pots $(12 \mathrm{~cm}$ in diameter and $11 \mathrm{~cm}$ in height) containing a mixture of $90 \%$ loam soil and $10 \%$ organic compost. When plants reached the third true leaf stage, they were infested with aphids and watered as required.

\subsection{Biological and Life Table Parameters Determination}

For each cultivar, nearly 50-60 apterous females from the stock colonies maintained on each host plant for at least 3 generations were placed individually into individual plant conditioned into cages especially designed and tested, according to [15]. This method is a suitable alternative to clip cages. After $24 \mathrm{~h}$, the adult and all but one 
first-instar were removed, and all single first instars were the cohorts on each cultivar $(n \approx 45)$. The whole assay was carried out in a growth chamber at $20 \pm 1^{\circ} \mathrm{C}$, about $70 \% \mathrm{RH}$ and $14 \mathrm{~h}$ photophase. Individual aphids were checked daily for ecdysis and survivorship. The exuviae presence was used to define instar changes. After the immature became an adult, mortality and fecundity were checked daily, and offspring were counted and removed from each cage until the death of the adult. When necessary, the adults were transferred to another plant of the same cultivar. The study was completed when the last female died. The mean and standard error of mean (SEM) of nymph developmental, pre-reproductive, reproductive, post-reproductive duration time and longevity, as well as life table construction and calculation of demographic and mortality parameters were developed by using the specific computer program JLIFETABLE [16]. JLIFETABLE's outputs also include the SEM estimate of all life table and mortality statistics by using the Jackknife procedure $[17,18]$. The equations to obtain the life table and mortality parameters followed [19,20] and [21] and are shown in Table 1.

\subsection{Data Analysis}

Data were prior tested for normality using the Shapiro - Wilk test in PROC UNIVARIATE and tested for homogeneity of variances using the Levene test in PROC GLM of SAS program [22]. Data were not normally distributed, and the SAS macro multiple comparison Kruskal - Wallis nonparametric ANOVA based was used to compare all biological attributes and life table parameters [23]. The nymphal mortality percentages were analyzed with Fisher's Exact test in PROC FREQ [22]. Variations in fecundity over time for a specific cultivar were analyzed as a fourth-order polynomial regression with fecundity as the dependent variable and cultivar and age as discrete independent variables. Cultivar effects were analyzed by using leastsquare means to adjust for the polynomial age effects. Means of fecundity were then compared among cultivars, and means were separated with option of Tukey - Kramer test in the GLM. The survival data recorded on the various cultivars was analyzed in two ways as follows: nonparametric comparison of survival curves in PROC LIFETEST and estimation of Gompertz and Weibull models parameters with nonlinear regression in PROC NLIN [22] and the respective coefficient of determination of nonlinear regression like $R^{2}$ (Pseudo- $R^{2}$ ) were manually obtained with the expression: Pseudo- $R^{2}=1-$ SS (Residual) / SS (Total Corrected) [24]. The probability that an individual lives at least to age $x$ was calculated by the following equations:

\section{Gompertz}

$$
S_{x}=e^{\left[\left(\frac{a}{b}\right)\left(1-e^{b x}\right)\right]}
$$

where $a$ is the initial mortality rate and $b$ is the exponential rate of increase in the death rate.

Weibull

$$
S_{x}=e^{-\left(\frac{x}{b}\right)^{c}}
$$

for $x>0$, where $b$ is a scale parameter that is inversely related to the mortality rate and $c$ is a shape parameter that allows the model to

\begin{tabular}{|c|c|c|}
\hline Parameter & Units & Equation \\
\hline Intrinsic rate of natural increase $\left(r_{m}\right)$ & 우이우./day & $\sum_{x=0}^{\infty} l_{x} m_{x} e^{-r_{m} t}=1$ \\
\hline Net Reproductive rate $\left(R_{0}\right)$ & 우이우.generation & $R_{0}=\sum \mathrm{I}_{\mathrm{x}} \mathrm{m}_{\mathrm{x}}$ \\
\hline Mean generation time $(T)$ & Days & $T=\frac{\sum \mathrm{xl}_{\mathrm{x}} \mathrm{m}_{\mathrm{x}}}{\sum \mathrm{m}}$ \\
\hline $\begin{array}{l}\text { Finite rate of increase }(\lambda) \\
\text { Doubling time (DT) }\end{array}$ & $\begin{array}{l}1 / \text { day } \\
\text { Days }\end{array}$ & $\begin{array}{l}\lambda=e^{r_{m}} \\
D=\underline{\ln 2}\end{array}$ \\
\hline Entropy $(\mathrm{H})$ & Days & $H=\frac{\sum_{x=0}^{m} \mathrm{~d}_{\mathrm{x}} \mathrm{e}_{\mathrm{x}}}{\mathrm{e}_{0}}$ \\
\hline Average daily mortality $(\bar{\mu})$ & Days & $\mu=\frac{1}{\mathrm{e}_{0}}$ \\
\hline
\end{tabular}

Table 1. Demographic and mortality parameters used with its respective units and equations

References: $x=$ age,$I_{x}=$ age-specific survival, $m_{x}=$ daily fecundity, $d_{x}=$ frequency of deaths $e_{x}=$ life expectancy, $e_{0}=$ total life expectancy or life expectancy at day 0 
produce survival distributions of different forms $[25,26]$. Values of the shape parameter $c>1,=1$, or $<1$ correspond to Deevey's type I, II, or III survivorship curves, respectively.

\section{RESULTS}

\subsection{Development of Immature Stages}

Differences in the duration of each instar were always significant among the cohorts reared on the seven cultivars $(P<.0001)$ (Table 2$)$. There were significant differences in the time of the immature development among the cohorts on different cultivars (Kruskal - Wallis $H=258.88$; $d f=6 ; P<.0001 ;$ Table 2). Individuals fed on Biolnta 1002 and Buck Meteoro developed significantly faster than those on any other cultivars, while those on ACA 315, followed by Baguette 12 P, Biolnta 2004 and Klein Yarará, have longer development time (Table 2). The incidence of cultivar particularly on the relative span of $3^{\text {rd }}$ and $4^{\text {th }}$ instar appears to have a close relationship with complete development time. Mortality at the nymphal stage was always below $10 \%$ in all tested cultivars and no significant differences were observed (Fisher's exact test, $P=.1197)$.

\subsection{Reproductive Periods and Adult Longevity}

The mean pre-reproductive period was always below $1 \mathrm{~d}$. This is because, in many cases, we have found the exuviae, the adult and some offspring in a day. When the $4^{\text {th }}$ nymph became adult and it had offspring in less than 1 day, the duration of pre-reproductive period became zero. However, there are significant differences in the span of pre-reproductive period (Kruskal - Wallis $H=24.02 ; d f=6 ; P<.0001$ ) and it was shorter on the Baguette $12 \mathrm{P}$ and Biolnta 1002 cultivars (Table 3). The wheat cultivars used in this study had a noticeable effect on the reproductive period of $R$. padi (Kruskal - Wallis $H=202.05$; $d f$ $=6 ; P=.0001 ;$ Table 3$)$. The reproductive period was longer for adults reared on cv. Biolnta 1002 followed by Biolnta 2004 and ACA 315. The post-reproductive period was shorter on Buck Meteoro and LE 2330 (Table 3). Adult longevity varied significantly among cultivars (Kruskal Wallis $H=205.04 ; d f=6 ; P<.0001$, Table 3 ), and it was the longest on Biolnta 1002 and followed by two groups that can be clearly distinguished; the first, with relatively short-lived aphids reared on Baguette 12 P, Buck Meteoro, Klein Yarará and LE 2330, and the second, with the relatively long-lived aphids reared on the remaining cultivars (Table 3 ).

\subsection{Survival Rate and Fecundity}

The log-rank test used to compare age-specific survival curves has shown significant differences among cohorts reared on different cultivars (logrank test $\left.X^{2}=322.24 ; d f=6 ; P<.0001\right)$. The survival curves of aphids reared on cv. Biolnta 2004 was the most diverse compared to

Table 2. Development time in days (Mean (SEM)) and mortality of immatures of $R$. padi on seven wheat ( $T$. aestivum) cultivars

\begin{tabular}{|c|c|c|c|c|c|c|c|}
\hline Cultivar & $\mathbf{N}$ & $\begin{array}{l}\text { Nymphal } \\
\text { mortality (\%) }\end{array}$ & $\begin{array}{l}1^{\text {st }} \\
\text { instar }\end{array}$ & $\begin{array}{l}2^{\text {nd }} \\
\text { instar }\end{array}$ & $\begin{array}{l}3^{\text {rd }} \\
\text { instar }\end{array}$ & $\begin{array}{l}4^{\text {th }} \\
\text { instar }\end{array}$ & $\begin{array}{l}\text { Combined } \\
\text { nymphs }\end{array}$ \\
\hline ACA 315 & 55 & 8.33 & $\begin{array}{l}2.7 a^{*} \\
(0.24)\end{array}$ & $\begin{array}{l}2.4 \mathrm{a} \\
(0.22)\end{array}$ & $\begin{array}{l}2.5 \mathrm{a} \\
(0.23)\end{array}$ & $\begin{array}{l}2.3 a \\
(0.25)\end{array}$ & $\begin{array}{l}9.9 a \\
(0.14)\end{array}$ \\
\hline Baguette $12 \mathrm{P}$ & 58 & 3.33 & $\begin{array}{l}2.5 \mathrm{a} \\
(0.22)\end{array}$ & $\begin{array}{l}2.2 a b \\
(0.19)\end{array}$ & $\begin{array}{l}2.1 \mathrm{a} \\
(0.21)\end{array}$ & $\begin{array}{l}2.1 \mathrm{a} \\
(0.17)\end{array}$ & $\begin{array}{l}8.9 a b \\
(0.12)\end{array}$ \\
\hline Biolnta 1002 & 44 & 4.36 & $\begin{array}{l}1.2 \mathrm{c} \\
(0.15)\end{array}$ & $\begin{array}{l}1.7 c \\
(0.17)\end{array}$ & $\begin{array}{l}1.3 \mathrm{~b} \\
(0.18)\end{array}$ & $\begin{array}{l}1.4 \mathrm{c} \\
(0.22)\end{array}$ & $\begin{array}{l}5.7 \mathrm{~d} \\
(0.11)\end{array}$ \\
\hline Biolnta 2004 & 44 & 2.22 & $\begin{array}{l}2.0 \mathrm{~b} \\
(0.24)\end{array}$ & $\begin{array}{l}2.1 a b \\
(0.12)\end{array}$ & $\begin{array}{l}2.1 \mathrm{a} \\
(0.19)\end{array}$ & $\begin{array}{l}2.4 \mathrm{a} \\
(0.31)\end{array}$ & $\begin{array}{l}8.6 \mathrm{~b} \\
(0.15)\end{array}$ \\
\hline Buck Meteoro & 46 & 9.80 & $\begin{array}{l}1.8 \mathrm{~b} \\
(0.22)\end{array}$ & $\begin{array}{l}1.7 c \\
(0.19)\end{array}$ & $\begin{array}{l}1.4 \mathrm{~b} \\
(0.21)\end{array}$ & $\begin{array}{l}1.6 \mathrm{c} \\
(0.20)\end{array}$ & $\begin{array}{l}6.6 \mathrm{~d} \\
(0.11)\end{array}$ \\
\hline Klein Yarará & 54 & 0.00 & $\begin{array}{l}1.9 \mathrm{~b} \\
(0.10)\end{array}$ & $\begin{array}{l}1.4 \mathrm{~d} \\
(0.18)\end{array}$ & $\begin{array}{l}1.6 \mathrm{~b} \\
(0.20)\end{array}$ & $\begin{array}{l}1.8 \mathrm{bc} \\
(0.28)\end{array}$ & $\begin{array}{l}8.8 \mathrm{~b} \\
(0.14)\end{array}$ \\
\hline LE 2330 & 47 & 9.62 & $\begin{array}{l}1 . .9 \mathrm{~b} \\
(0.13)\end{array}$ & $\begin{array}{l}1.8 \mathrm{bc} \\
(0.18)\end{array}$ & $\begin{array}{l}1.4 \mathrm{~b} \\
(0.19)\end{array}$ & $\begin{array}{l}2.2 \mathrm{ab} \\
(0.23)\end{array}$ & $\begin{array}{l}7.3 c \\
(0.10)\end{array}$ \\
\hline KW H & & & 168.80 & 102.66 & 132.99 & 75.01 & 258.88 \\
\hline & & & 6 & 6 & 6 & & 6 \\
\hline $\mathrm{P}$ & & & $<.0001$ & $<.0001$ & $<.0001$ & $<.0001$ & $<.0001$ \\
\hline
\end{tabular}


Table 3. Reproductive periods and longevity in days (Mean (SEM)) of $R$. padi on seven wheat ( $T$. aestivum) cultivars

\begin{tabular}{lllll}
\hline Cultivar & $\begin{array}{l}\text { Pre- } \\
\text { reproductive }\end{array}$ & Reproductive & $\begin{array}{l}\text { Post- } \\
\text { reproductive }\end{array}$ & Longevity \\
\hline ACA 315 & $0.51 \mathrm{a}^{*}$ & $28.05 \mathrm{~b}$ & $4.71 \mathrm{a}$ & $43.24 \mathrm{~b}$ \\
& $(0.09)$ & $(1.54)$ & $(0.64)$ & $(1.76)$ \\
Baguette 12 P. & $0.11 \mathrm{~d}$ & $12.00 \mathrm{c}$ & $1.66 \mathrm{c}$ & $(1.18)$ \\
& $(0.09)$ & $(0.94)$ & $(0.51)$ & $47.36 \mathrm{~b}$ \\
Biolnta 1002 & $0.21 \mathrm{C}$ & $34.97 \mathrm{ab}$ & $3.29 \mathrm{ab}$ & $(1.56)$ \\
& $(0.06)$ & $(1.41)$ & $(0.41)$ & $67.41 \mathrm{a}$ \\
Biolnta 2004 & $0.14 \mathrm{~d}$ & $54.84 \mathrm{a}$ & $3.80 \mathrm{a}$ & $(3.17)$ \\
& $(0.05)$ & $(2.98)$ & $(0.49)$ & $19.96 \mathrm{c}$ \\
Buck Meteoro & $0.33 \mathrm{~b}$ & $11.96 \mathrm{c}$ & $1.07 \mathrm{c}$ & $(1.56)$ \\
& $(0.07)$ & $(1.34)$ & $(0.35)$ & $28.11 \mathrm{c}$ \\
Klein Yarará & $0.48 \mathrm{a}$ & $17.83 \mathrm{c}$ & $3.02 \mathrm{~b}$ & $(1.49)$ \\
& $(0.07)$ & $(1.15)$ & $(0.54)$ & $26.02 \mathrm{c}$ \\
LE 2330 & $0.26 \mathrm{C}$ & $16.43 \mathrm{c}$ & $1.98 \mathrm{c}$ & $(1.81)$ \\
& $(0.06)$ & $(1.47)$ & $(0.46)$ & 205.04 \\
KW H & 24.02 & 202.05 & 50.552 & 6 \\
df & 6 & 6 & 6 & $<.0001$ \\
$\mathrm{P}$ & .0005 & .0001 & $<01$ & $<.05)$
\end{tabular}

the remaining cultivars (Table 4, Fig. 1). No significant differences were observed among the survival curves on Biolnta 1002, Buck Meteoro, Klein Yarará and LE 2330 and there were also no significant differences between ACA 315 and Baguette 12 P. (Table 4, Fig. 1).

Table 4. Multiple comparison log-rank test of survival rate curves of $\boldsymbol{R}$. padi on seven wheat ( $T$. aestivum) cultivars

\begin{tabular}{ll}
\hline Cultivar & Log-rank value \\
\hline ACA 315 & $-11.93 \mathrm{~b}^{*}$ \\
Baguette 12 P. & $-23.88 \mathrm{~b}$ \\
Biolnta 1002 & $30.90 \mathrm{C}$ \\
Biolnta 2004 & $-72.30 \mathrm{a}$ \\
Buck Meteoro & $31.24 \mathrm{C}$ \\
Klein Yarará & $23.40 \mathrm{C}$ \\
LE 2330 & $22.57 \mathrm{c}$ \\
\hline${ }^{*}$ Cultivars and log-rank statistic followed by the same \\
letter have not significantly difference between aphid \\
survival curves (Adjustment for multiple comparison log- \\
$\quad$ rank test, Tukey - Kramer correction; $\alpha=.05$ )
\end{tabular}

The daily fecundity patterns observed over lifespan of the aphids were best described by polynomial regression and were not a linear function of age, forming a more or less skewedright pattern (Fig. 1). The regression analysis (Table 5) showed a very strong effect of host plants on the aphid daily fecundity, even after variation due to age was removed $(F=9.95$; $d f=$ $6,317 ; P<.0001)$. The highest numbers were recorded from 8 to 40 days after birth, with values of $3.2,4.1,2.4,2.9,3.5,3.0$ and 3.5 nymphs per day on cultivars ACA 315, Baguette 12 P, Biolnta 1002, Biolnta 2004, Buck Meteoro, Klein Yarará and LE 2330, respectively. Overall, the number of nymphs per female per day on Baguette $12 \mathrm{P}$ was higher than that on the other six cultivars.

\subsection{Life Table Parameters}

There were significant differences in the life expectancy at birth $\left(\mathrm{e}_{0}\right)$ of $R$. padi (Kruskal Wallis $H=168.66 ; d f=6 ; P<.0001)$. Life expectancy was shorter on Baguette $12 \mathrm{P}$, Buck Meteoro, Klein Yarara and LE 2330 (19 to 29 days). The longest $e_{0}$ was observed on $\mathrm{cv}$. Biolnta 2004 (66.83 days). On the other cultivars, $\mathrm{e}_{0}$ varied from 40 to 47 days (Table 6 ).

The entropy $(\mathrm{H})$ and the average daily mortality parameter $\bar{\mu}$ of $R$. padi reared on the seven wheat cultivars also showed significant differences, Kruskal - Wallis $H=34.79$; $d f=6$; $P=$ .0001 and Kruskal - Wallis $H=130.20$; $d f=6$; $P=$ .0001 , respectively (Table 6 ). The entropy values suggested that the survival schedule of $R$. padi was convex $(\mathrm{H}<0.5)$ on all cultivars, except on Buck Meteoro and LE 2330, which was slightly concave or straight $(\mathrm{H}=0.51)$ (Table 5, Fig. 1). The average daily mortality of $R$. padi was on its lowest level on Biolnta 2004 ( $\bar{\mu}=0.01511$ days) while $\bar{\mu}$ ranged from 0.02133 to 0.05137 days on the remaining cultivars (Table 6). 
Table 5. Polynomial regression analysis of daily fecundity on aphid age and cultivar

\begin{tabular}{|c|c|c|c|c|c|}
\hline Source & df & Mean square & $\mathbf{F}$ & $\mathbf{P}$ & $\mathbf{R}^{2}$ \\
\hline Model & 17 & 13.0335059 & 51.01 & $<.0001$ & 0.711862 \\
\hline Mean (Age) & 1 & 57.57841279 & 225.35 & $<.0001$ & \\
\hline Mean $^{2}$ & 1 & 6.37888886 & 24.97 & $<.0001$ & \\
\hline Mean $^{3}$ & 1 & 27.63701134 & 108.16 & $<.0001$ & \\
\hline Mean $^{4}$ & 1 & 29.35857215 & 114.9 & $<.0001$ & \\
\hline Cultivar & 6 & 2.54183293 & 9.95 & $<.0001$ & \\
\hline Age $\times$ Cultivar & 7 & 10.57682932 & 41.39 & $<.0001$ & \\
\hline Error & 317 & 0.2555105 & & & \\
\hline \multicolumn{6}{|c|}{ Daily fecundity least square means } \\
\hline \multicolumn{2}{|r|}{ Cultivar } & \multicolumn{3}{|l|}{ L.S.M \pm S.E.M. } & \\
\hline & ACA 315 & \multicolumn{2}{|c|}{$1.26652752 \pm 0.06816773 c^{*}$} & & \\
\hline & Baguette $12 \mathrm{P}$ & \multicolumn{2}{|c|}{$1.33916023 \pm 0.06951779 \mathrm{c}$} & & \\
\hline & BiolNTA 1002 & \multicolumn{2}{|c|}{$2.61271608 \pm 0.29889037 \mathrm{a}$} & & \\
\hline & BiolNTA 2004 & \multicolumn{2}{|c|}{$1.62336243 \pm 0.06037396 b$} & & \\
\hline & Buck Meteoro & \multicolumn{2}{|c|}{$0.25911679 \pm 0.18978813 \mathrm{e}$} & & \\
\hline & Klein Yarará & \multicolumn{2}{|c|}{$0.1789009 \pm 0.12597915 \mathrm{e}$} & & \\
\hline & LE 2330 & \multicolumn{2}{|c|}{$0.48805672 \pm 0.1324186 \mathrm{~d}$} & & \\
\hline
\end{tabular}
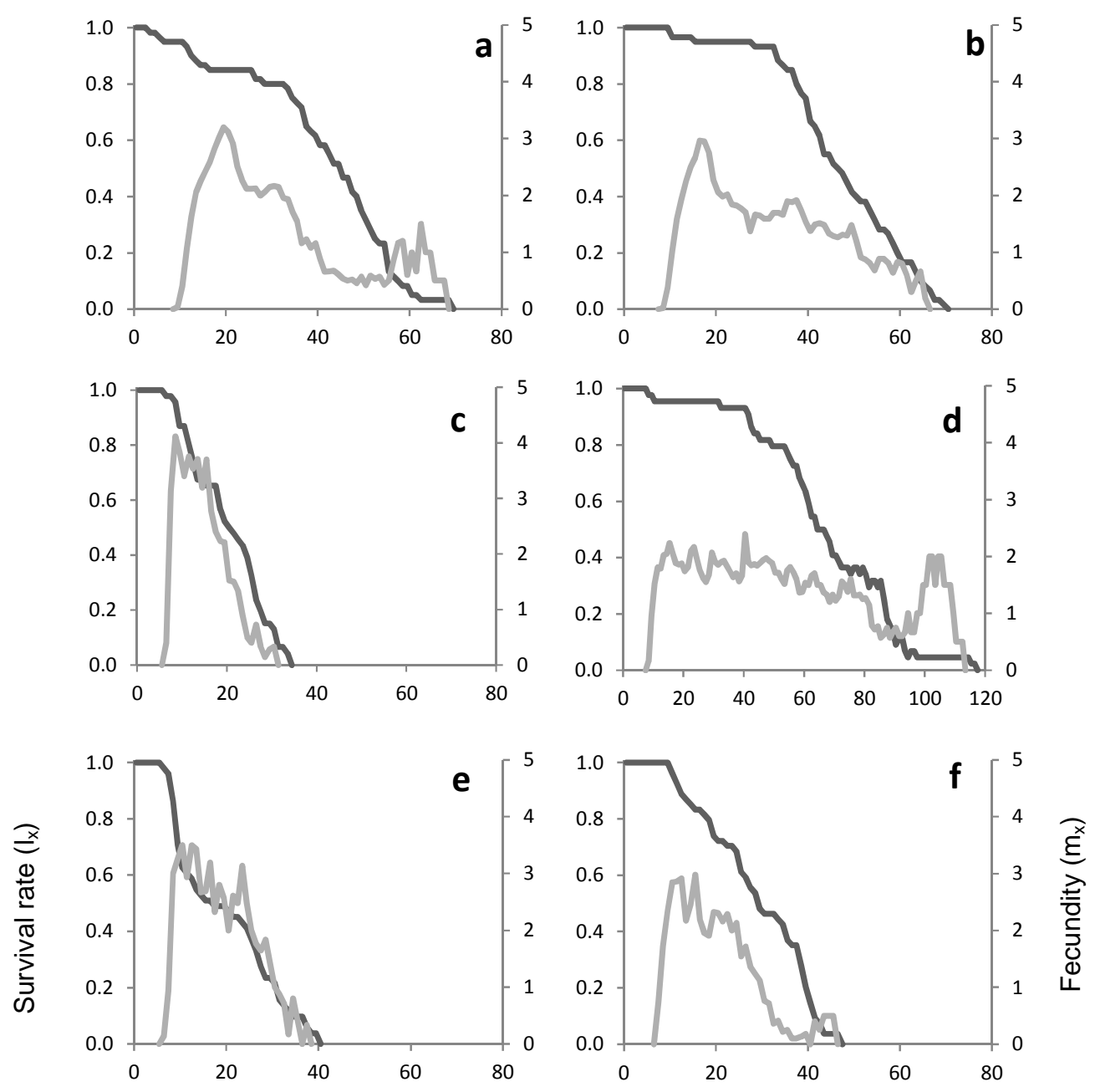


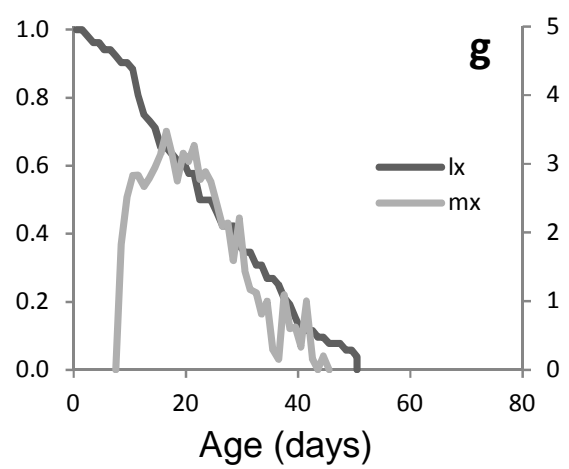

Fig. 1. Age-specific survival rate $\left(\mathrm{I}_{\mathrm{x}}\right)$ and fecundity $\left(\mathrm{m}_{\mathrm{x}}\right)$ of $R$. padi reared on different wheat $(T$. aestivum) cultivars. a: ACA315, b: Baguette 12 9., c: Biolnta 1002, d: Biolnta 2004, e: Buck Meteoro, f: Klein Yarará, g: LE 2440

The $r_{m}$, net reproductive rate $\left(R_{0}\right)$, mean generation time $(T)$, finite rate of increase (ë), and population doubling time (DT) were calculated for aphids developing on seven wheat cultivars (Table 6). There were significant differences in $r_{m}$ values among the populations on the seven cultivars (Kruskal - Wallis $\mathrm{H}=$ 158.08; $d f=6 ; P<.0001)$. The lower $r_{m}$ values for $R$. padi were observed on ACA 315, Baguette 12 $P$ and Biolnta 2004 (0.204-0.221 females.female ${ }^{1}$.day ${ }^{-1}$ ). The $\mathrm{R}_{0}$ values of $R$. padi estimated on wheat cultivars ranged from 30.78 to 98.98 females.female ${ }^{-1}$.generation ${ }^{-1}$, with significant differences among cultivars (Kruskal-Wallis $H=$ 114.75; $d f=6 ; P<.0001)$. The populations reared on cv. Buck Meteoro had a lower $\mathrm{R}_{0}$ value (30.78 females.female . generation $^{-1}$ ) but it is not significantly different than those reared on Biolnta 1002, Klein Yarará and LE 2330. There were significant differences in $\mathrm{T}$ values among the populations reared on the seven cultivars
(Kruskal-Wallis $H=271.89 ; d f=6 ; P<.0001$ ) and ranged from 10.91 to 20.78 days (Table 6). Significant differences in the finite rate of increase $(\lambda)$ (Kruskal-Wallis $H=154.67 ; d f=6$; $P<.0001)$ and doubling times (DT) (KruskalWallis $H=170.68 ; d f=6 ; P<.0001)$ were found for $R$. padi populations reared on the tested wheat cultivars. The last mentioned life table parameter (DT) varied from 3.40 days on cv. ACA 315 to 2.12 days on Biolnta 1002 (Table 6).

\subsection{Mortality Models}

Parameters of nonlinear regression analysis between survival rate and age of $R$. padi reared on different wheat cultivars using the Gompertz and Weibull models are showed in Table 7. A significant fit was obtained between the survival rate and age of $R$. padi reared on wheat cultivars using these models $(P<.01)$.

Table 6. Life table parameters of $R$. padi reared on different wheat (T. aestivum) cultivars (Means (SEM))

\begin{tabular}{lllllllll}
\hline Cultivar & $\mathbf{r}_{\mathbf{m}}$ & $\mathbf{R}_{0}$ & $\mathbf{T}$ & $\boldsymbol{\Lambda}$ & $\mathbf{D T}$ & $\mathbf{H}$ & $\overline{\boldsymbol{\mu}}$ & $\mathbf{e}_{\mathbf{0}}$ \\
\hline ACA 315 & $0.204 \mathrm{c}^{*}$ & $54.63 \mathrm{~b}$ & $19.66 \mathrm{a}$ & $1.226 \mathrm{c}$ & $3.40 \mathrm{a}$ & $0.32 \mathrm{~b}$ & $0.0250 \mathrm{bc}$ & $40.67 \mathrm{~b}$ \\
& $(0.004)$ & $(6.26)$ & $(0.43)$ & $(0.005)$ & $(0.05)$ & $(0.04)$ & $(0.00099)$ & $(3.39)$ \\
Baguette 12 P & $0.219 \mathrm{c}$ & $64.49 \mathrm{~b}$ & $19.00 \mathrm{a}$ & $1.245 \mathrm{c}$ & $3.16 \mathrm{a}$ & $0.25 \mathrm{~b}$ & $0.0213 \mathrm{c}$ & $46.57 \mathrm{~b}$ \\
& $(0.007)$ & $(2.73)$ & $(0.20)$ & $(0.010)$ & $(0.05)$ & $(0.04)$ & $(0.00311)$ & $(1.20)$ \\
Biolnta 1002 & $0.327 \mathrm{a}$ & $35.32 \mathrm{c}$ & $10.91 \mathrm{c}$ & $1.387 \mathrm{a}$ & $2.12 \mathrm{c}$ & $0.37 \mathrm{~b}$ & $0.0504 \mathrm{a}$ & $19,72 \mathrm{c}$ \\
& $(0.007)$ & 3.76 & $(0.33)$ & $(0.009)$ & $(0.07)$ & $(0.05)$ & $(0.00304)$ & $(1.87)$ \\
Biolnta 2004 & $0.221 \mathrm{c}$ & $98.98 \mathrm{a}$ & $20.78 \mathrm{a}$ & $1.247 \mathrm{c}$ & $3.13 \mathrm{a}$ & $0.30 \mathrm{~b}$ & $0.0151 \mathrm{~d}$ & $66.86 \mathrm{a}$ \\
& $(0.004)$ & $(3.64)$ & $(0.30)$ & $(0.005)$ & $(0.07)$ & $(0.04)$ & $(0.00129$ & $(2.09)$ \\
Buck Meteoro & $0.272 \mathrm{~b}$ & $30.78 \mathrm{c}$ & $12.61 \mathrm{bc}$ & $1.313 \mathrm{~b}$ & $2.54 \mathrm{~b}$ & $0.51 \mathrm{a}$ & $0.0513 \mathrm{a}$ & $19.13 \mathrm{c}$ \\
& $(0.009)$ & $(3.67)$ & $(0.34)$ & $(0.012)$ & $(0.09)$ & $(0.04)$ & $(0.00414)$ & $(1.52)$ \\
Klein Yarará & $0.273 \mathrm{~b}$ & $39.06 \mathrm{c}$ & $13.45 \mathrm{~b}$ & $1.313 \mathrm{~b}$ & $2.54 \mathrm{~b}$ & $0.33 \mathrm{~b}$ & $0.0352 \mathrm{~b}$ & $28.61 \mathrm{c}$ \\
& $(0.006)$ & $(2.33)$ & $(0.24)$ & $(0.007)$ & $(0.05)$ & $(0.04)$ & $(0.00199)$ & $(1.49)$ \\
LE2330 & $0.259 \mathrm{~b}$ & $39.59 \mathrm{c}$ & $14.22 \mathrm{~b}$ & $1.296 \mathrm{~b}$ & $2.67 \mathrm{~b}$ & $0.51 \mathrm{a}$ & $0.0411 \mathrm{~b}$ & $24.40 \mathrm{c}$ \\
& $(0.003)$ & $(3.37)$ & $(0.27)$ & $(0.004)$ & $(0.04)$ & $(0.02)$ & $(0.00096)$ & $(1.75)$ \\
\hline
\end{tabular}

*Life table parameters followed by the same letter are not significantly different ( $M C-K W \alpha=.05)$ 
Table 7. Estimated parameters (SE) of nonlinear regression between survival rate and age $\boldsymbol{R}$. padi reared on different wheat ( $T$. aestivum) cultivars fitted to Gompertz and Weibull models

\begin{tabular}{lllllll}
\hline Cultivars & \multicolumn{3}{c}{ Gompertz } & \multicolumn{3}{c}{ Weibull } \\
\cline { 2 - 7 } & $\mathbf{a}$ & $\mathbf{b}$ & $\mathbf{R}^{2}$ & $\mathbf{b}$ & $\mathbf{c}$ & $\mathbf{R}^{2}$ \\
\hline ACA 315 & $2.13 \mathrm{E}-03$ & $7.35 \mathrm{E}-02$ & 0.9898 & 47.8825 & 3.3490 & 0.9750 \\
Baguette 12 P & $(1.66 \mathrm{E}-04)$ & $(2.23 \mathrm{E}-03)$ & & $(0.4326)$ & $(0.1384)$ & \multirow{2}{*}{0.9930} \\
& $9.94 \mathrm{E}-04$ & $8.41 \mathrm{E}-02$ & 0.9883 & 52.2701 & 4.0675 & 0.9930 \\
Biolnta 1002 & $(1.04 \mathrm{E}-04)$ & $(2.62 \mathrm{E}-03)$ & & $(0.2202)$ & $(0.0933)$ & \multirow{2}{*}{0.9851} \\
& $8.75 \mathrm{E}-03$ & $1.09 \mathrm{E}-01$ & 0.9863 & 23.2169 & 2.4658 & \\
Biolnta 2004 & $(8.50 \mathrm{E}-04)$ & $(5.98 \mathrm{E}-03)$ & & $(0.2756)$ & $(0.1053)$ & \\
& $1.12 \mathrm{E}-03$ & $5.00 \mathrm{E}-02$ & 0.9898 & 75.3224 & 3.5027 & 0.9919 \\
Buck Meteoro & $(8.10 \mathrm{E}-05)$ & $(1.30 \mathrm{E}-03)$ & & $(0.3237)$ & $(0.0708)$ & \\
\multirow{2}{*}{ Klein Yarará } & $2.17 \mathrm{E}-02$ & $5.45 \mathrm{E}-02$ & 0.9477 & 22.5350 & 1.6217 & 0.9606 \\
& $(2.68 \mathrm{E}-03)$ & $(8.21 \mathrm{E}-03)$ & & $(0.5215)$ & $(0.0954)$ & \\
LE2330 & $4.71 \mathrm{E}-03$ & $8.65 \mathrm{E}-02$ & 0.9873 & 33.5425 & 2.7186 & 0.9810 \\
& $(4.04 \mathrm{E}-04)$ & $(3.61 \mathrm{E}-03)$ & & $(0.3514)$ & $(0.1117)$ & \\
& $1.48 \mathrm{E}-02$ & $4.93 \mathrm{E}-02$ & \multirow{2}{*}{0.9930} & 28.7410 & 1.7783 & 0.9928 \\
& $(6.04 \mathrm{E}-04)$ & $(2.09 \mathrm{E}-03)$ & & $(0.2327)$ & $(0.0392)$ & \\
\hline
\end{tabular}

\section{DISCUSSION}

Plant species vary in their suitability as a host for insects. Such variation in host suitability can be shown by measuring insect performance and preference on different host species [27]. It can also be shown at the cultivar level of the same plant species since cultivars differ in their chemical and morphological characteristics, also influencing their suitability as hosts $[28,29,30]$. As a result, assessing the resistance of different cultivars to pest insects can provide valuable information on their suitability as hosts. In the current study, we have demonstrated that the biology of $R$. padi was significantly influenced by the wheat cultivars tested.

The survival rate of the aphid displayed different trends on the seven wheat cultivars, with longevity and life expectancy at birth being lower on Baguette 12 P, Buck Meteoro, Klein Yarara and LE 2330. The entropy parameter provides a useful summary measure for characterizing differences in the shape of survival curves among cohorts [21]. Survival curves for aphids reared on any cultivar followed the Deevey's type I survival curve, as "c" values of the Weibull model were higher than one. Deevey's type I survival curve is convex, but it was slightly concave or straight for the aphids reared on Buck Meteoro and LE 2330 as shown by the entropy $(\mathrm{H})$. However, the parameter "c" of the Weibull model for these cultivars was closest to one than the remaining values; it is not clearly detected by the model. On the other hand, the parameter "b" of the Weibull model is inversely related to the mortality rate. This value was lower on Biolnta 1002 and Buck Meteoro, corresponding to a higher average daily mortality $(\bar{\mu})$ on these cultivars. In the Gompertz model, the initial rate of mortality (a) and the exponential rate of increase in the death rate (b) were higher on Buck Meteoro and Biolnta 1002, respectively. Because of the observed significant fit, both models are valid to explain the survival curves of aphids reared on all tested cultivars.

Despite the low life expectancy, low net reproductive rate and high mortality, the bird cherry - oak aphid, at least for the population used in our study, performed significantly better on Biolnta 1002. This was expressed as a high population increase and decreased nymphal duration. On Biolnta 1002, the $r_{m}$ value was the highest because the population reared on this cultivar had short immature development and one of the lowest mean generation time values. On the other hand, aphids reared on Biolnta 2004 had the highest $R_{0}$ value, however, the $r_{m}$ value was one of the lowest and had a relatively long immature development. This is due to a very high $\mathrm{R}_{0}$ in combination with a long $\mathrm{T}$, and this contributes to a depressed $r_{m}$ value. On Biolnta 1002 , R. padi can increase its generations in a given time; while on Biolnta 2004, even increasing the offspring per generation is not enough to increase the $r_{m}$ value. Overall, our results demonstrate that the ACA 315, Baguette $12 \mathrm{P}$ and, relatively Biolnta 2004 cultivars, are less suitable hosts for the development of $R$. padi compared to the other cultivars examined. Aphids reared on ACA 315, Baguette $12 \mathrm{P}$, Biolnta 2004 and Klein Yarará had a longer developmental time and the mean generation time (T) was also longer except on Klein Yarará. Under natural conditions, longer generation and 
developmental time will result in a slower population growth on these cultivars, increasing the exposure of the herbivore to natural enemies [31]. This is likely to occur particularly on Biolnta 2004. The shorter developmental times were observed on Biolnta 1002 and Buck Meteoro but the highest intrinsic rate of natural increase $\left(r_{\mathrm{m}}\right)$ and finite rate of increase $(\lambda)$ were observed on the former, which led to a higher performance of the aphid on this cultivar.

Measuring life table parameters, especially the intrinsic rate of natural increase $\left(r_{m}\right)$, is the best way to evaluate insect performance on host plants [32]. In the present study, $r_{m}$ ranged from 0.327 to 0.204 females/female/day on the wheat cultivars tested and was highest on Biolnta 1002 and lower on ACA 315, Baguette $12 \mathrm{P}$ and Biolnta 2004. These values are close to that estimated for $R$. padi on wheat cv.Dragón $\left(r_{m}=\right.$ 0.263 females/female/ day) [33] and on different cereal crops, including wheat of the Biolnta cultivar family $\left(r_{m}=0.238\right.$ females/female/day $)$ [4]. In this last case, the entire experiment was conducted at $2^{\circ} \mathrm{C}$ while we have used a temperature $4^{\circ} \mathrm{C}$ lower. It is remarkable that the low intrinsic rates of natural increase reported by [34] for $R$. padi reared on ten wheat cultivars at ${ }^{20} \mathrm{C}$, including Buck Meteoro, ranged from 0.22 to 0.17 females/female/day, without significant differences between them. It is also interesting that a short nymphal period, reproductive period and longevity was reported on Buck Meteoro, of 2.88, 4.88 and 8.44 days, respectively, while we observed 6.6, 11.96 and 19.96 days, respectively in the current study. The reasons for these differences are unknown, but could be because they are two different aphid populations or due to unnoticed details in the methodology used.

Although the nature of the possible mechanisms for antibiosis wasnot studied in our experiment, it may be involved with the low performance observed for $R$. padi on ACA 315 and Baguette 12 P. Perhaps Biolnta 2004 should be considered a partially resistant cultivar due to its higher longevity, net reproductive rate and life expectancy, but it may also enhance the effectiveness of natural enemies. Buck Meteoro, Klein Yarará and LE 2440 could be considered relatively intermediate in resistance to the aphid. Knowledge of the degree of cultivar susceptibility or resistance and the biology of a pest is a fundamental component of an IPM program and can inform the detection and monitoring of pest infestations, cultivar selection and crop improvement. Hence, our findings may provide important information for designing a comprehensive program for IPM of $R$. padi in Argentina.

\section{CONCLUSION}

Among tested cultivars, ACA 315 and Baguette $12 \mathrm{P}$ can be considered resistant to $R$. padi, Biolnta 2004 should be considered a partially resistant and Biolnta 1002 could be considered susceptible. Buck Meteoro, Klein Yarará and LE 2440 could be considered relatively intermediate in resistance.

\section{COMPETING INTERESTS}

Authors have declared that no competing interests exist.

\section{REFERENCES}

1. Blackman RL, Eastop VF. Aphids on the world's crops. An Identification Guide. $2^{\text {nd }}$ ed. Chichester, Wiley Ltd.; 2000.

2. Blackman RL, Eastop VF. Taxonomic Issues. In van Emden $\mathrm{H}$, Harrington $\mathrm{R}$, editors. Aphids as crop pests. WallingFord, CABI Publishing; 2007.

3. Hesler L. Resistance to Rhopalosiphum padi (Homoptera: Aphididae) in three Triticale accessions. J Econ Entomol. 2005;98(2):603-610.

4. Descamps LR, Sánchez Chopa C. Population growth of Rhopalosiphum padi L. (Homoptera: Aphididae) on different cereal crops from the Semiarid Pampas of Argentina under laboratory conditions. Chilean Journal of Agricultural Research. 2011;71(3):390-394.

5. Savaris M, Lampert S, Salvadori JR, Lau D, Pereira PRVS, Smaniotto MA. Population growth and damage caused by Rhopalosiphum padi (L.) (Hemiptera, Aphididae) on different cultivars and phenological stages of wheat. Neotropical Entomology. 2013;42(5):539-543.

6. Imwinkelried J, Frana J, Salto C. Plagas Animales. In: Enfermedades, malezas y plagas animales del cultivo de trigo. Acintacnia- Cuaderno Técnico. 1988; 8(27):30-34. Spanish.

7. Medina-Ortega KJ, Bosque-Pérez NA, Ngumbi E, Jiménez-Martínez ES, Eigenbrode S. Rhopalosiphum padi (Hemiptera: Aphididae) responses to volatile cues from Barley yellow dwarf virus-infected wheat. Environ Entomol. 2009;38(3):836-45. 
8. Mohammad N. An overview of the IPM techniques for saving the agricultural biodiversity in Malaysia. Journal of Applied Science and Agriculture. 2014;9(6):26662670.

9. Hesler L, Riedell W, Kieckhefer R, Haley S. Responses of Rhopalosiphum padi (Homoptera: Aphididae) on cereal aphidresistant wheat accessions. J Agric Urban Entomol. 2002;19(3):133-140.

10. Smith, CM. Plant resistance to arthropods: Molecular and conventional approaches. Dordrecht. The Netherlands. Springer; 2006.

11. Hosseini-Tabesh B, Sahragard A, KarimiMalati A. A laboratory and field condition comparison of life table parameters of Aphis gossypii Glover (Hemiptera: Aphididae). Journal of Plant Protection Res. 2015;55(1):1-7.

12. Soufbaf M, Fathipour Y, Karimzadeh J, Zalucki MP. Development and age-specific mortality of diamondback moth on Brassica host plants: Pattern and cause of mortality under laboratory conditions. Ann Entomol Soc Am. 2010;100:574-579.

13. Hafiz NA. Use of life tables to asses host plant resistance in cowpea to Aphis craccivora Koch (Homoptera: Aphididae). Ass. Univ. Bull. Environ. Res. 2006;9(1): 1-6.

14. Golizadeh A, Esmaeili N, Razmjou J, Rafiee-Dastjerdi H. Comparative life tables of the potato tuber worm, Phthorimaea operculella, on leaves and tubers of different potato cultivars. Journal of Insect Science. 2014;14(42):1-11.

15. Giudici AC, La Rossa FR. Evaluación de una metodología de crianza individual de áfidos alternativa a la jaula de aplique Libro de Resúmenes. XV Jornadas Fitosanitarias Argentinas. Santa $\mathrm{Fe}$, Argentina. 7-9 October. 2015;294. Spanish.

16. La Rossa FR. Nuevo programa informático para la construcción de tablas de vida y la estimación de parámetros biológicos y poblacionales en insectos. Libro de resúmenes IX Congreso Argentino de Entomología. Posadas, Misiones, Argentina, 19-22 May. 2015;281. Spanish.

17. Meyer JS, Ingersoli CG, Mc Donalds LL, Boyce MS. Estimating uncertainty in population growth rates: Jackknife versus Bootstrap techniques. Ecology. 1986;67: 1156-1166.
18. Sokal RR, Rohlf FJ. Biometry: The principles and practice of statistics in biological research. $3^{\text {rd }}$ ed. New York. W. H. Freeman; 1995.

19. Birch LC. The intrinsic rate of natural increase of an insect population. J Anim Ecol. 1948;17:15-26.

20. Southwood RRE, Henderson PA Ecological Methods, $3^{\text {rd }}$ ed. Oxford. Blackwell Science; 2000.

21. Carey JR. Insect biodemography. Ann Rev Entomol. 2001;46:79-110.

22. SAS (®) Institute Inc. Help and Documentation, Cary, NC. USA; 2002.

23. Elliott AC, Hynan LS. A SAS(®) macro implementation of a multiple comparison post hoc test for a Kruskal - Wallis analysis. Comput Methods Prog Biomed. 2011;102(1):75-80.

24. Schabenberger $O$. Introduction to SAS. UCLA: Statistical Consulting Group (no date).

(Accessed: 28 November 2015)

Available:http://www.ats.ucla.edu/stat/sas/ notes2/

25. Deevey ES. Life tables for natural populations of animals. Q Rev Biol. 1947; 22:283-314.

26. Pinder JE III, Wiener JG, Smith MH. The Weibull distribution: $A$ new method of summarizing survivorship data. Ecology. 1978;59:175-179.

27. Frei A, Gu H, Bueno JM, Cardona C, Dorn S. Antixenosis and antibiosis of common beans to Thrips palmi Karny (Thysanoptera: Thripidae). J Econ Entomol. 2003; 93:1577-1584

28. Le Roux V, Dugravot S, Campan E, Dubois F, Vincent C, Giordanengo P. Wild Solanum resistance to aphids: Antixenosis or antibiosis? J. Econ. Entomol. 2008; 101(2):584-591.

29. Davis JA, Radcliffe EB, Ragsdale DW. Resistance to green peach aphid, Myzus persicae (Sulzer), and potato aphid, Macrosiphum euphorbiae (Thomas) in potato cultivars. Am. J. Potato Res. 2007; 84:259-269.

30. Áhman I, Glinwood R, Ninkovic V. The potential for modifying plant volatile composition to enhance resistance to arthropod pests. Plant Science Reviews. 2010;2010:1-10.

31. Thomson LJ, Macfadyen S, Hoffmann AA. Predicting the effects of climate change on natural enemies of agricultural pests. Biological Control. 2010;52:296-306. 
32. Razmjou J, Moharramipour S, Fathipour Y, Mirhoseini SZ. Effect of cotton cultivar on performance of Aphis gossypii (Homoptera: Aphididae) in Iran. J Econ Entomol. 2006;99:1820-1825.

33. La Rossa FR, Vasicek A, Mendy P, Moreno Kiernan A, Paglioni A. Biología y demografía de Diuraphis noxia (Mordv.), Rhopalosiphum padi (L.) y Metopolophium dirhodum (Wlk.) sobre trigo en condiciones de laboratorio (Hemiptera: Aphididae). Boletín de la Sociedad Entomológica Aragonesa. 2005;36:227-231. Spanish.

34. Sánchez Chopa C, Descamps LR. Reproducción de Rhopalosiphum padi (Hemiptera, Aphididae) en cultivares de trigo en la región del sudoeste bonaerense de Argentina. Acta Entomológica Chilena. 2013;33(12):17-22. Spanish.

(C) 2017 La Rossa et al.; This is an Open Access article distributed under the terms of the Creative Commons Attribution License (http://creativecommons.org/licenses/by/4.0), which permits unrestricted use, distribution, and reproduction in any medium, provided the original work is properly cited.

Peer-review history:

The peer review history for this paper can be accessed here:

http://sciencedomain.org/review-history/19755 